\title{
Evaluation of Stat5 as a potential drug target in bcr/abl-induced leukemias
}

\author{
Andrea Hölbl ${ }^{1}$, Christian Schuster ${ }^{1}$, Boris Kovacic ${ }^{2}$, Maria Hölzl1 , \\ Sabine Fajmann ${ }^{1}$, Florian Grebienn ${ }^{3}$, Lothar Hennighausen ${ }^{4}$, Yongzhi Cui ${ }^{4}$, \\ Richard Moriggl ${ }^{5}$, Hartmut Beug ${ }^{2}$ and Veronika Sexl*1
}

\begin{abstract}
Address: ${ }^{1}$ Centre of Biomolecular Medicine and Pharmacology, Medical University of Vienna, 1090 Vienna, Austria, ${ }^{2}$ Institute of Molecular Pathology (IMP), 1030 Vienna, Austria, ${ }^{3}$ Research Center for Molecular Medicine of the Austrian Academy of Sciences (CeMM), 1090 Vienna, Austria, ${ }^{4}$ NIDDK, National Institute of Health, Bethesda, MD 20892, USA and ${ }^{5}$ Ludwig-Boltzmann Institute for Cancer Research (LBI-CR), 1090 Vienna, Austria

Email: Veronika Sexl* - veronika.sexl@meduniwien.ac.at

* Corresponding author
\end{abstract}

from 14th Scientific Symposium of the Austrian Pharmacological Society (APHAR)

Innsbruck, Austria. 21-22 November 2008

Published: 5 November 2008

BMC Pharmacology 2008, 8(Suppl I):A4 doi:I0.1 186/I47I-22I0-8-SI-A4

This abstract is available from: http://www.biomedcentral.com/I47I-22 I0/8/SI/A4

(c) $2008 \mathrm{Hölbl} \mathrm{et} \mathrm{al;} \mathrm{licensee} \mathrm{BioMed} \mathrm{Central} \mathrm{Ltd.}$

The Stat 5 transcription factors Stat5a and Stat5b have been implicated in lymphoid development and transformation. Using the complete Stat 5 knockout mice, we have previously shown that $S t a t 5 a / b^{\text {null } / \text { null }}$ cells were resistant to transformation and leukemia development induced by Abelson oncogenes, whereas Stat $5 a / b^{\Delta N / \Delta N}$ cells readily transformed. So far, these findings showed distinct susceptibility to Abelson-induced transformation of Stat $5 a /$ $b^{\Delta N / \Delta N}$ and Stat $5 a / b^{\text {null } / \text { null }}$ mice and defined Stat 5 as key regulator of initial transformation. In this study, we tested whether Stat $5 \mathrm{a} / \mathrm{b}$ is also essential for the maintenance of a transformed state. Therefore we developed a system, where Stat $5 \mathrm{a} / \mathrm{b}$ could be deleted at will. Abelson-transformed B lymphoid cells were generated from Stat $5 a / b f l / f l$ gene targeted mice that had been crossed with Mx-Cre transgenic animals. These leukemic Stat5a/bfl/fl MxCre cells were then used to test effects of Stat $5 \mathrm{a} / \mathrm{b}$ ablation in vitro and in vivo. In vitro, Stat5a/b deletion resulted in a cell cycle arrest followed by apoptosis. Nine days after deletion, no viable cells could be detected. In line with that, a down-regulation of Stat 5 target genes mediating G1/S transition within the cell cycle and viability, such as cyclin D2 and cyclin D3, c-myc and bcl- $\mathrm{x}_{\mathrm{L}}$ was found. When leukemic Stat $5 a / b f l / f l M x$ Cre cells were injected into wild type or immuno-compromised mice leukemia rapidly developed. Again, deletion of Stat5a/b in vivo within the leukemic cells significantly counteracted disease progression as indicated by an increase of leukemia latency from 16 to 49 days. Eventually, all animals succumbed to a Stat5a/b-positive leukemia indicating that a few residual cells escaped deletion. Moreover, p53 abruption or overexpression of the oncogene did not alter the susceptibility to Stat 5 loss of established leukemic cell lines. Taken together our data define a key role for Stat $5 \mathrm{a} / \mathrm{b}$ not only for lymphoid development but also for lymphoid transformation. Stat $5 \mathrm{a} / \mathrm{b}$ is necessary for the initial transformation as well as for leukemia progression. This absolute necessity for the proliferation and viability of Abelsontransformed cells puts Stat5a/b into the spotlight of new therapeutic strategies for the treatment of $b c r / a b l$-induced leukemias. 